\title{
STOCHASTIC AND DETERMINISTIC BEHAVIOURS OF 316 STAINLESS STEEL PITTING CORROSION IN PHOSPHATE-BORATE BUFFER CONTAINING SODIUM CHLORIDE
}

\author{
R. C. Salvarezza, N. de Cristofaro, ${ }^{*}$ C, Pallotta ${ }^{\dagger}$ and A. J. Arvia \\ Instituto de Investigaciones Fisicoquímicas Té́ricas y Aplicadas (INIFTA), Facultad de Ciencias \\ Exactas, Universidad Nacional de La Plata, Casilla de Correo 16, Sucursal 4, $1900 \mathrm{La} \mathrm{Plata,} \mathrm{Argentina}$
}

(Received 27 August 1986)

\begin{abstract}
The pitting corrosion of 316 stainless steel (SS) in phosphate-borate buffer (pH 8.00) containing $0.5 \mathrm{M} \mathrm{NaCl}$ at $25^{\circ} \mathrm{C}$ is studied through current transients run under a constant potential step, potentiodynamic runs and scanning electron microscopy. According to the potential and time windows different laws for pitting kinetics are observed. For small number of pits, the kinetics of pit growth is governed by a stochastic law whereas for a large number of pits a deterministic law which corresponds to an instantaneous nucleation and growth is obeyed. At the initial stages two different rate constants for pit nucleation are obtained. This fact correlates with two well distinguishable types of pits observed through SEM observations. Results are discussed through a model involving the penetration of chloride ions through the passive film and the existence of at lcast two types of sites at the metal surface which in turn become centers of pit nucleation. Pit stability is directly related to the $\mathrm{Cr}$ (III) content in the passive layer which can be shifted by changing the applied potential with respect to that of the $\mathrm{Cr}_{2} \mathrm{O}_{3} / \mathrm{CrO}_{4}^{2-}$ redox couple.
\end{abstract}

\section{INTRODUCTION}

The initiation of localized corrosion has been considered as a rare event both in space and time and consequently it can be treated as a statistical phenomenon[1-4]. Current bursts associated with pit activation (birth) and pit repassivation (death) are observed during potentiodynamic and potentiostatic experiments [5,6]. Birth processes can be analyzed in terms of a nucleation rate, whereas death processes are accounted for by the probability of pit dying. Potential dependent nucleation rates derived from induction times for pit initiation as a statistical variable are given in the literature[1, 2]. On the other hand, death processes are related to the ability of the passive layer for self repairing. The latter process for stainless steel depends on both water $[7,8]$ and chromium content in the passive film which in turn change with the applied potential and temperature[9]. Recently a potential dependent nucleation rate for stable pits, a potential independent rate for unstable pit formation and a potential independent probability for pit dying could be distinguished from experiments run within a relatively narrow potential window [4], although no correlation between those parameters with the number of pits and pit morphology from microscopic examination was established. In this respect one could expect a great influence of the applied potential on the number of centres for pit growth so that the transition from stochastic or deterministic models for the kinctics of

* Departamento de Electroquímica, Instituto Nacional de Tecnología Industrial (INTI).

tDepartamento de Fisicoquimica, Facultad de Ciencias Exactas y Naturales, Universidad de Buenos Aires. pitting processes could change according to the selected potential window.

This work deals with the localized corrosion of 316 SS in a strong buffered chloride ions-containing medium, studied by using potentiodynamic and potentiostatic techniques complemented with scanning electron microscopy observations. Special attention is paid to the influence of the applied potential on the early stages of the nucleation and growth of stable pits with the purpose of establishing the condition for application of either a stochastic or a deterministic model to interpret pitting kinetics.

\section{EXPERIMENTAL}

Working electrodes were made of $316 \mathrm{SS}$ rods axially embedded in Araldite holders to obtain circular exposed areas of $0.2 \mathrm{~cm}^{2}$ geometric area. Occasionally, iron electrodes (Johnson Matthey, $0.5 \mathrm{~mm}$ dia. rod) prepared as described in [9] were used. Firstly, the metal surface was mechanically polished with fine grained emery paper followed with alumina paste (1 $\mathrm{mm}$ dia.) on polishing cloth to obtain a mirror surface. Each polished specimen was rinsed with twice distilled water and dried in air at room temperature. A new pretreated specimen was used for each run.

Measurements were made at $25^{\circ} \mathrm{C}$ with a conventional Pyrex glass cell containing the electrolyte solution $(0.2 \mathrm{l})$. The potential of the working electrode was measured against a saturated calomel electrode (sce) provided with a Luggin-Haber capillary tip. All potentials in the text are referred to the sce. The electrolyte prepared from A.R. chemicals and bidistilled water consisted of $0.10 \mathrm{M} \mathrm{K} \mathrm{H}_{2} \mathrm{PO}_{4}+0.05 \mathrm{M} \mathrm{Na} \mathrm{B}_{4} \mathrm{O}$, (pH 8.00) containing $0.5 \mathrm{M} \mathrm{NaCl}$. Previously to each 
run the solution was purged with purified nitrogen during $3 \mathrm{~h}$. Polarization curves [apparent current density $(j)$ vs potential $(E)]$ were run by applying to the specimen a singular triangular potential sweep (STPS) between the cathodic $\left(E_{\mathrm{s}, \mathrm{c}}\right)$ and the anodic $\left(E_{\mathrm{s}, \mathrm{a}}\right)$ switching potential at different scan rate (v). Potentiostatic current transients were recorded after applying to the specimen a potential program which included successively a cathodization at $E_{\mathrm{c}}=-1.10 \mathrm{~V}$ for $90 \mathrm{~s}$ to electroreduce the metal surface, an anodization at $E_{\mathrm{a}}=-0.50 \mathrm{~V}$ for $90 \mathrm{~s}$ to form a prepassive layer, and finally, a potential step to $E_{\mathrm{s}}$. For each $E_{\mathrm{s}}$, 40-80 measurements were made.

Induction times $\left(t_{\mathrm{j}}\right)$ were obtained from the current transients at constant potential. Due to the ambiguity to define $t_{\mathrm{i}}$ by setting an arbitrary threshold current value to be exceeded, only $t_{\mathrm{i}}$ values for stable pit growth were considered. These values were taken as the last time at which the current shows a low value similar to that recorded for the completely passive specimen. This mcans that for any time, $t$, greater than $t_{\mathrm{i}}$ the specimen is not able to repassivate. Complementary SEM micrographs were taken by using a Philips 500 electron scunning microscope.

\section{RESULTS}

\section{Voltammetric results}

Voltammograms resulting for $316 \mathrm{SS}$ specimens in phosphate-borate buffer run at $0.02 \mathrm{~V} \mathrm{~s}^{-1}$ between $E_{\mathrm{s}, \mathrm{c}}=-1.20 \mathrm{~V}$ and $E_{\mathrm{s}, \mathrm{a}}=1.00 \mathrm{~V}$ (Fig. 1) show three anodic current peaks at $-0.60 \mathrm{~V}$ (peak $\mathrm{A}_{\mathrm{I}}$ ), at $-0.30 \mathrm{~V}$ (peak $A_{11}$ ) and at $0.70 \mathrm{~V}$ (peak $A_{I I t}$ ) (Fig. 1). The returning scan presents two cathodic current peaks one located at $0.1 \mathrm{~V}$ (peak $\mathrm{C}_{1 \mathrm{II}}$ ) and another one at $-0.50 \mathrm{~V}$ (peak $C_{11}$ ). Peak $A_{I}$ can be assigned to the electroformation of a $\mathrm{Fe}(\mathrm{OH})_{2}$ layer on the pre-existing $\mathrm{Cr}_{2} \mathrm{O}_{3}$ layer[10]. The hydrous $\mathrm{Fe}(\mathrm{OH})_{2}$ film is electro-oxidized to hydrous FeOOH at potentials corresponding to current peak $A_{I I}$ yielding a complex film of inner $\mathrm{Cr}_{2} \mathrm{O}_{3}$ and outer FeOOH[10, 11]. Peak $A_{I I I}$ was associated with the electro-oxidation of $\mathrm{Cr}$ (III) to $\mathrm{Cr}$ (VI) into the passive film and to the formation of soluble $\mathrm{CrO}_{4}^{2-}[10]$. The electroreduction of $\mathrm{Cr}$ (VI) in the film to $\mathrm{Cr}$ (III) was related to peak $\mathrm{C}_{11}$ while the broad peak $C_{1 I}$ was assigned to the electroreduction of the hydrous

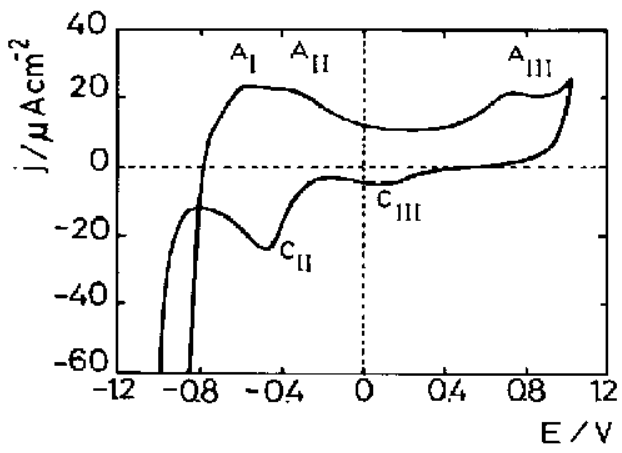

Fig. 1. $j / E$ profile in phosphate-borate buffer, $\mathrm{pH}=8.00 ; 0$ $=0.02 \mathrm{~V} \mathrm{~s}^{-1}, E_{\mathrm{s}, \mathrm{c}}=-1.10 \mathrm{~V} ; E_{\mathrm{s}, \mathrm{a}}=1.0 \mathrm{~V}$.

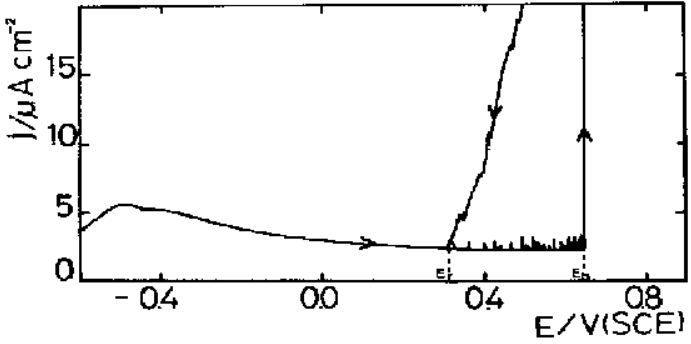

Fig. 2. $j / E$ profile in phosphate-borate buffer $+0.5 \mathrm{M} \mathrm{NaCl}$, $\mathrm{pH}=8.00 ; v=0.0002 \mathrm{Vs}^{-1}$, from $E_{s, \mathrm{c}}=-1.10 \mathrm{~V}$ to $E_{\mathrm{b}}$ and then returning to the repassivation potential $E_{\mathbf{r}}$.

FeOOH film $[11,12]$. The $j / E$ profiles recorded in the phosphate borate buffer containing $0.5 \mathrm{M} \mathrm{NaCl}$ show similar current peaks although when the potential exceeds a certain critical value $\left(E_{b}\right)$ a remarkable increase in current due to the initiation of pitting corrosion can be observed (Fig. 2). Current instabilities related to breakdown and repair events at the passive film are detected in the potential range preceeding $E_{\mathrm{b}}$. The returning scan exhibits a considerable hysteresis loop which extends from $E_{\mathrm{b}}$ to a potential $E_{\mathrm{r}}$ located at ca $0.30 \mathrm{~V}$. At $E_{\mathrm{r}}$ the passive film has been fully restored. Similar runs made with pure iron specimens exhibit the initiation of pitting at lower potential and the current instabilities prior to $E_{\mathrm{b}}$ are practically absent.

\section{Potentiostatic current transients}

The current transients were run at constant potential values ranging from $-0.30 \mathrm{~V}$ to $0.70 \mathrm{~V}$. Each current transient was preceeded by the following electrochemical pretreatment. The specimen was firstly held at $-1.10 \mathrm{~V}$ for $90 \mathrm{~s}$ to achieve a reproducible electroreduced surface and later stepped to $-0.5 \mathrm{~V}$ to form the prepassive layer. According to the value of $E_{8}$, different current transients can be obtained (Fig. 3). Thus, for $E_{\mathrm{s}}<0.30 \mathrm{~V}$, the current transient decreases continuously resembling the characteristics of already known passive metal surfaces (Fig. 3a). In this case the current transient is mainly determined by the growth of the passive film and the simultaneous electrodissolution of the metal through it [13]. Otherwise, for $E_{\mathrm{s}}$ set in the $0.30-0.50 \mathrm{~V}$ range different current transients can be obtained for experiments performed under nominally jelentical conditions. For a rational analysis, these transients can be divided into two groups, one of them showing a behaviour similar to that described for those resulting at $E_{\mathrm{s}}<0.30 \mathrm{~V}$ and another one exhibiting an initially decreasing current which reaches a minimum value and afterwards a remarkable increasing one. The current minimum determines an induction time $\left(t_{\mathrm{j}}\right)$ for stable pit initiation and the increase in current is associated with stable pit growth. However, in many cases stable pitting is not achieved and in this case a considerable number of fluctuations in the current is seen. Some runs exhibit a rather abrupt decrcase in current to attain a valuc similar to that observed in passive specimens (Fig. 3b). Current fluctuations are also recorded during both the induction period and the rising part of the current transients 


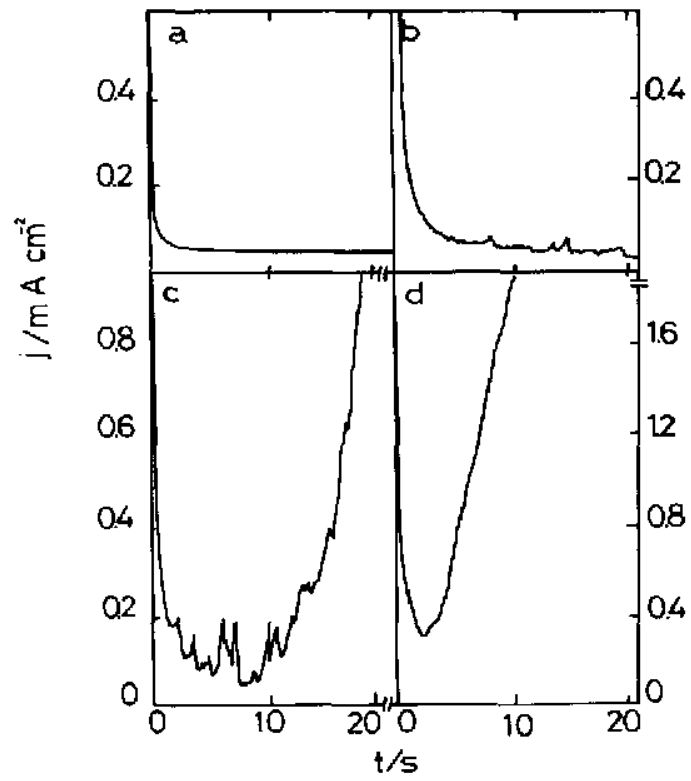

Fig. 3. Current transients at constant potential $\left(E_{s}\right)$ in phosphate-borate buffer $+0.5 \mathrm{M} \mathrm{NaCl}, \mathrm{pH}=8.00$. After cathodizing $90 \mathrm{~s}$ at $E_{\mathrm{c}}=-1.10 \mathrm{~V}$, another $90 \mathrm{~s}$ at $E_{\mathrm{a}}=$ $-0.50 \mathrm{~V}$, and finally stepped to $E_{\mathrm{y}}$, (a) $E_{\mathrm{s}}<0.30 \mathrm{~V}$, (b) and (c) $0.30 \leqslant E_{\mathrm{s}} \leqslant 0.50 \mathrm{~V}$, (d) $E_{\mathrm{s}}>0.50 \mathrm{~V}$.

associated with pit growth (Fig. 3c). The number of specimens presenting stable pitting increases according to $E_{\mathrm{s}}$. For $E_{\mathrm{s}}>0.5 \mathrm{~V}$, the corresponding current transients become progressively better defined, more reproducible and practically noiseless (Fig. 3d). The probability, $P_{n \geq 1}$, to form at least one stable pit[14] can be evaluated from the current transients resulting for different $E_{\mathrm{s}}$ (Fig. 4). The value of $P_{n \geqslant 1}$ increases according to $E_{\mathrm{s}}$ and for $E_{\mathrm{s}}>0.5 \mathrm{~V}, P_{n \geqslant 1} \rightarrow 1$. Therefore, as $E_{\mathrm{s}}$ increases, the survival time of specimens is drastically reduced (Fig. S, a and b). Finally for the current transients, the rising part of the current

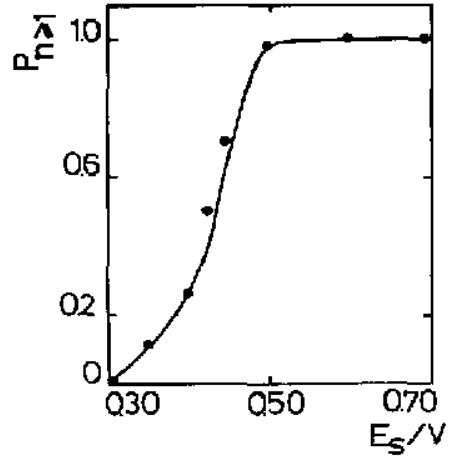

Fig. 4. $P_{n \neq 1}$ us $E$, plots.

resulting for $E_{\mathrm{s}}>0.50 \mathrm{~V}$ obeys a linear $j v s t^{2}$ relationship (Fig. 6).

\section{SEM observations}

SEM micrographs of surfaces resulting from anodizing in the $0.30<E_{s}<0.50 \mathrm{~V}$ range $\left(t_{\mathrm{s}}=210 \mathrm{~s}\right)$ show for many specimens a single large pit on the surface (Fig. 7a), where others present a small number of pits with a broad distribution in size. The number of pits increases according to $E_{\mathrm{s}}$ and for $E_{\mathrm{s}}>0.60 \mathrm{~V}$ a great number of pits with radii as large as $50 \mu \mathrm{m}$ can be observed (Fig. 7b). Furthermore, the SEM micrographs also shown that a large number of pits of smaller size are also present together with large pits (Fig. 7, b and c). These results suggest that the two different types of pits either involve a long time for initiation or the corresponding growth rates are substantially different. Likewise for $E_{\mathrm{s}}>0.6 \mathrm{~V}$, the number of large pits becomes independent of the anodizing time. The mean number $\langle n\rangle$ and standard deviation, $\sigma$, for large pits resulting at different $E_{\mathrm{s}}$ and $t_{\mathrm{s}}=210 \mathrm{~s}$ are assembled in Table 1. It can be noticed that $\sigma /\langle n\rangle$ ratio decreases as $E_{\mathrm{s}}$ increases. Preliminary results show that the increase either in $E_{\mathrm{a}}$ or in the anodization time at constant $E_{\mathrm{s}}$ reduces strongly the $\langle n\rangle$ values.

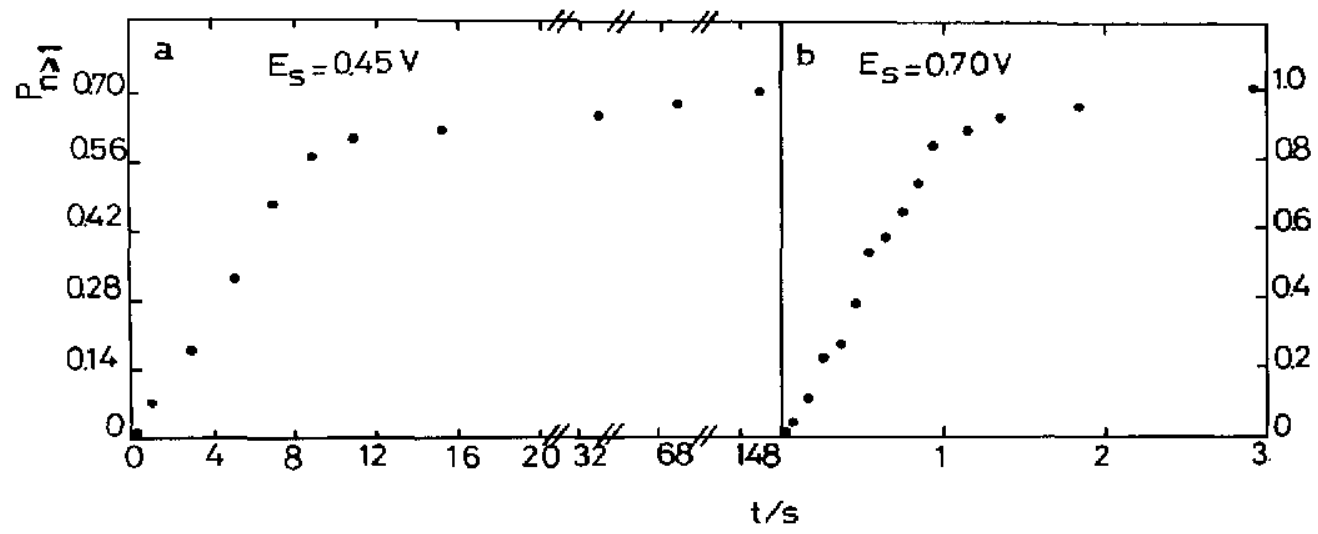

Fig. 5. $P_{n \geqslant 1}$ vs \& plots for different $E_{\mathrm{s}}$ values. (a) $E_{\mathrm{s}}=0.45 \mathrm{~V}$, (b) $E_{\mathrm{s}}=0.70 \mathrm{~V}$. 


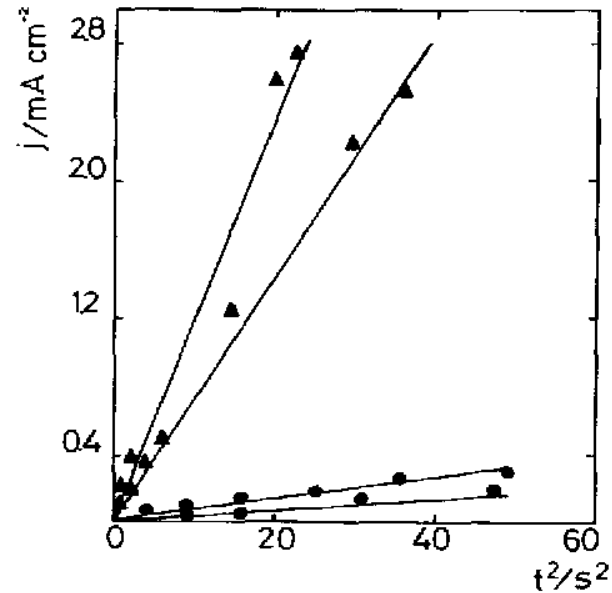

Fig. 6. $j$ bs $t^{2}$ plots for different $E_{s}$ values. (e) $E_{\mathrm{s}}=0.60 \mathrm{~V}$, (A) $E_{\mathrm{s}}=0.70 \mathrm{~V}$.

\section{DISCUSSION}

\section{The stochastic range for pitting of 316 SS}

The pitting corrosion of $316 \mathrm{SS}$ in neutral buffered media containing chloride ions is a complex process involving passive film breakdown, pit propagation and pit repassivation. Further understanding of the initial stage of the mechanism of pitting can be attempted through the analysis of a large number of potentiostatic current transients. In principle, both the shupe of the current transients and the voltammetric loop resulting in the pitting corrosion domain for $316 \mathrm{SS}$ resembles those for localized corrosion of other metals and alloys in aqueous environment containing chloride[13]. Nevertheless, $316 \mathrm{SS}$ in these aggressive media as compared to other metals exhibits a rather unusual behaviour because most of the samples are in fact not corroding but become passive and stable. This electrochemical behaviour is accompanied by the relative scattering in pitting parameters and strong current oscillations observed for $316 \mathrm{SS}$. It is also established that as $E_{\mathrm{s}}$ increases current fluctuations either disappear or become sufficiently small as compared to the mean transient current value that they are no longer distinguishable. As a first approach, the phenomenology of current fluctuations for 316 SS in chloridecontaining solutions can be described through recently stochastic models advanced for pitting corrosion of SS [4]. Accordingly each current burst can be associated with the birth and transient growth of a pit which can be followed by the abrupt stop of the process due to the death of the growing pit leading to repassivation. The birth and growth of a pit can be analyzed in terms of the rate of convertion of a surface site into a pit nucleation centre, whereas the death can be accounted for the probability of pit dying. Otherwise, when the current burst exceeds a given value, the system attains irreversibility the stable pitting domain. At this stage, the system has not self repassivation ability and as the activation process cannot be counterbalanced the first current burst becomes effective to produce stable pitting.

The rate of convertion of a surface site into a nucleation centre for stable pit growth, $\lambda$, can be estimated from the time dependence of the probability of formation of at least one nucleous for stable pit growth $\left(P_{n \geq 1}\right)$ at a constant potential by means of the Poisson distribution law. The expression for $\left(P_{n \geqslant 1}\right)$ corresponding to a stationary value of $\lambda$ is given by $[14]$ :

$$
P_{n \geqslant 1}=1.0-\exp (-\hat{a} t)
$$

According to equation (1), $\lambda$ can be estimated by plotting $\ln \left(1.0-P_{n \geq 1}\right)$ vs $t$ (Fig. 8) where the first term is the logarithm of the probability of maintaining the specimen out of stable pitting. In agreement with previous observations reported in the literature $[1,3]$ for the pitting corrosion of stainless steel in chloride solutions, these plots for different $E_{\mathrm{s}}$ values exhibit two reasonable linear portions with their corresponding slopes $\lambda_{1}$ and $\lambda_{2}$, respectively. The values of $\hat{\lambda}_{1}$ and $\lambda_{2}$ fit reasonable straight lines by plotting either $I_{n} \hat{\lambda}_{i} v s E_{\mathrm{s}}$ (Fig. 9) or ln $\lambda_{\mathrm{i}}$ vs $\left(E_{\mathrm{s}}-E_{\mathrm{b}}\right)^{-1}$, where $E_{\mathrm{b}}=0.30 \mathrm{~V}$ corresponds to the value of $E$ where $P_{n \geqslant 1}=0$ (Fig. 4). The dependence of $\lambda_{i}$ on potential could be interpreted, in principle, on the basis of either atomistic or classical simple nucleation models, through the evaluation of the number of atoms or molecules $\left(g^{*}\right)$ involved in the formation of a critical nucleous. The corresponding $g^{*}$ values from $\lambda_{i}$ leads to physically unrealistic figures, namely either to negative values for the atomistic model or to values smaller than one for classical models. Hence, the experimental results are inconsistent with simple nucleation models and the explanation of $\lambda_{i}$ values must be sought through another mechanistic approach for the early stage for pitting of $316 \mathrm{SS}$ in chloride-containing solutions.

A reasonable interpretation for $\lambda_{i}$ values can be based upon the passive film/anion interaction and the corresponding structure of the passivating layer. In this respect it should be noticed that there is a firm evidence for the penetration of chloride ions into the passive film of iron and SS as recently concluded by ESCA $[15]$ and SIMS[16] data. This fact suggests that $\lambda_{i}$ values could be relatcd to the time required for the aggressive anion to penetrate the entire passive film thickness to reach the metal surface instead of considering it as the time required to form a nucleous for stable pit growth. The new assumption is also consistent with linear $\ln \lambda_{\mathrm{j}}$ ws $E_{\mathrm{s}}$ relationships [17] but the high values of slopes can be related to the kinetics of the processes occurring at the passivating layer. The immediate conclusion from this interpretation is that the initial stage for stable pit formation should be controlled by the rate of penetration of the aggressive anion through the passive film and correspondingly the character istics of the passive film should play a substantial rolc in determining the $\lambda_{i}$ values.

It was suggested that both the rate of pit nucleation and current fluctuations recorded for a 240 grade polished $316 \mathrm{SS}$ in dilute sodium chloride are related to the production of persistent gradients of acidity at the scale of surface roughness of the metal[4]. However, this statement appears as a partial explanation of a more complex process as in the present case oscillations are also found in strong buffered solutions. The 

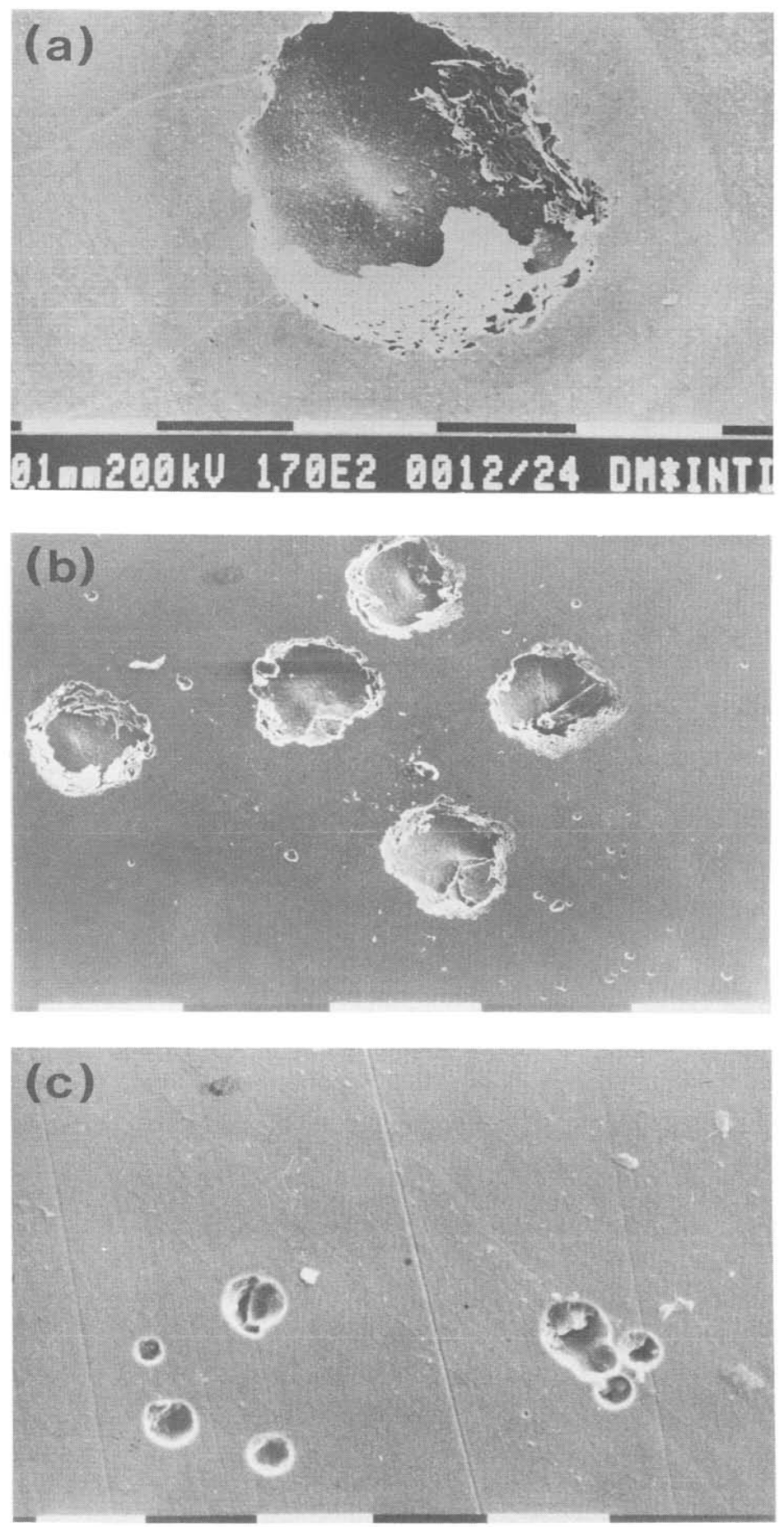

Fig. 7. SEM microphotographs obtained after anodizing a $316 \mathrm{SS}$ specimens during $210 \mathrm{~s}$ at different $E_{\mathrm{s}}$. (a) $E_{\mathrm{s}}=0.35 \mathrm{~V}$, scale $=0.1 \mathrm{~mm}$, (b) $E_{\mathrm{s}}=0.60 \mathrm{~V}$, scale $=0.1 \mathrm{~mm}$, (c) detail of (b), scale $=10 \mu \mathrm{m}$. 
Table 1. Number of pits resulting at different $E_{\mathrm{s}}$ values and $t_{\mathrm{s}}=210 \mathrm{~s}$

\begin{tabular}{crrl}
\hline$E_{\mathrm{s}}(\mathrm{V})$ & $\langle n\rangle$ & $\sigma$ & $\sigma /\langle n\rangle$ \\
\hline 0.45 & 2 & 2 & 1 \\
0.50 & 14 & 11 & 0.79 \\
0.60 & 25 & 19 & 0.76 \\
0.70 & 100 & 67 & 0.67 \\
\hline
\end{tabular}

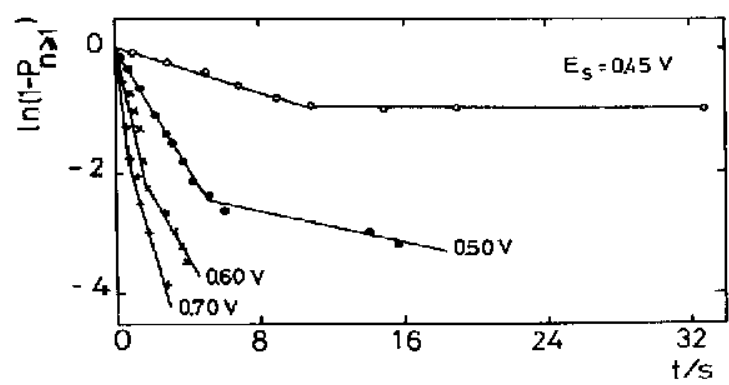

Fig. 8. In $\left(1-P_{n \geqslant 1}\right)$ ws $t$ plots for different $E_{\mathrm{s}}$ values.

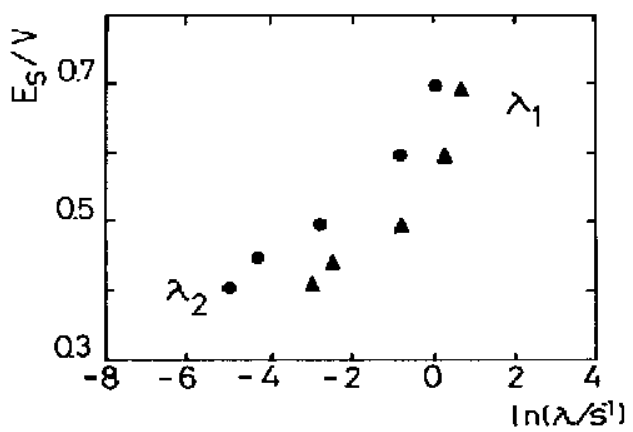

Fig. 9. $E_{\mathrm{s}}$ vs $\ln \lambda_{\mathrm{i}}$ plots.

repassivation ability of stainless steel depends on the content of both $\mathrm{Cr}$ (III) and water in the passive film [8, 9], and the corresponding breakdown potential and current oscillations are closely related to the chromium content in the passive film which in turn is determined by the electrode potential and temperature[9]. Likewise, the role of chromium in the self-repair ability of 316 SS was clearly demonstrated by using an iron electrode in chromate solutions containing chloride ions. These facts point out that the initiation and growth of pits at a constant potential should depend on the probability of pit dying, $\mu$ which is directly related to the chromium content in the passive layer[9]. The latter depends on potential through the following equilibrium at the electrochemical interface:

$$
\begin{gathered}
\mathrm{Cr}_{2} \mathrm{O}_{3}+5 \mathrm{H}_{2} \mathrm{O}=2 \mathrm{CrO}_{4}^{2-}+10 \mathrm{H}^{+}+6 e^{-}, \\
E_{\mathrm{r}}(\mathrm{pH} 7.0) / \mathrm{V}=0.37 .
\end{gathered}
$$

Accordingly, as $E_{8}$ becomes more positive the $\operatorname{Cr}(I I I)$ content in the passive film decreases and the ability of the film to self-repair diminishes. Hence, for 316 SS as the $\mathbf{C r}$ (III) content in the passive film decreases, $\mu$ should also decrease and the pit activation process continues without hinderance. Consequently, operating conditions can be accomplished where the first burst becomes effective for stable pitting corrosion.

The deterministic range for pitting of $316 \mathrm{SS}$

As $E_{\mathrm{s}}$ increases stable pitting nucleation cvents become gradually more frequent, that is $\lambda$ increases resulting in a large number of growing pits at the metal surface. Hence, at this stage each growing centre becomes less significant in relation to the total number of centres. Both progressive decrease in $\mu$ and the increase in $\lambda$ Iead to current transicnts with a gradual disappearance of oscillations. Consequently, beyond the transition range for $E_{\mathrm{s}}>0.50 \mathrm{~V}$, the kinetics of pitting can be described through a deterministic model, and in this case it is possible to derive some interesting information about the nucleation process involved in the pitting corrosion of $316 \mathrm{SS}$.

Let us start by assuming that pit growth involves a conical growth as it is approached by SEM micrographies and that the process undergoes under charge transfer control. Then, the apparent current density $\left(j_{\mathrm{s}}\right)$ is given by the expression [18]:

$$
\begin{aligned}
j_{\mathrm{s}} & =z F k_{3 \mathrm{c}}^{\cdot}\left[1.0-\exp \left(-\frac{\pi M^{2} k_{3 \mathrm{c}}^{2} N_{\mathrm{o}}\left(t-t_{\mathrm{i}}\right)^{2}}{\rho^{2}}\right)\right] \\
& =P_{1}\left[1.0-\exp \left(-P_{2}\left(t-t_{\mathrm{j}}\right)^{2}\right)\right]
\end{aligned}
$$

for instantaneous nucleation and,

$$
\begin{aligned}
j_{\mathrm{s}} & =z F k_{3 \mathrm{c}}^{\prime}\left[1.0-\exp \left(-\frac{\pi M^{2} k_{3 \mathrm{c}}^{2} N_{\mathrm{o}} i\left(t-t_{\mathrm{i}}\right)^{3}}{3 \rho^{2}}\right)\right] \\
& =P_{1}\left[1.0-\exp \left(-P_{2}^{\prime}\left(t-t_{\mathrm{i}}\right)^{3}\right)\right]
\end{aligned}
$$

for progressive nucleation where

$$
\begin{gathered}
P_{1}=z F k_{3 c}^{\prime} \\
P_{2}=\pi M^{2} k_{3 c}^{2} N_{0} / \rho^{2}
\end{gathered}
$$

and

$$
P_{2}^{\prime}=\pi M^{2} k_{3 \mathrm{c}}^{2} N_{\mathrm{o}} \lambda / 3 \rho^{2} .
$$

$k_{3 c}^{\prime}$ and $k_{3 c}$ are the rate constants perpendicular and parallel to the metal surface, respectively; $M$ is the molecular weight; $\rho$ is the density and $N_{0}$ is the number of sites available for the nucleation process.

Equations (3) and (4) for $\left(t-t_{j}\right) \rightarrow 0$ after serics expansions of the exponential terms lead to the following simplified cquations:

and

$$
j_{\mathrm{s}}=P_{1} P_{2}\left(t-t_{\mathrm{i}}\right)^{2}
$$

$$
j_{\mathrm{s}}=P_{1} P_{2}^{\prime}\left(t-t_{\mathrm{j}}\right)^{3} \text {. }
$$

For $\boldsymbol{E}_{5}>0.5 \mathrm{~V}$ the experimental results obey linear $j v \mathrm{~s}$ $t^{2}$ plots (Fig. 6). In principle, this result is consistent with an instantaneous nucleation of pits at the $316 \mathrm{SS}$ passivated surface, and it agrees with $\lambda$ values previously estimated. Accordingly, the number of sites converting into pits $(N)$ is related to $t$ through the equation [18]:

$$
N=N_{o}[1.0-\exp (-\lambda t)] .
$$

For $t=1 \mathrm{~s}$ and $\lambda_{1}$ values resulting for $E_{\mathrm{s}}=0.60 \mathrm{~V}$ and $E_{\mathrm{s}}=0.70 \mathrm{~V}$, respectively, equation (10) results:

$$
N \simeq N_{0} \text {. }
$$


The number of growing centres for an instantaneous nucleation which leads to a deterministic behaviour through reproducible practically fluctuation free current transients is about $80[19]$. This figure is approached for $E_{\mathrm{s}}>0.5 \mathrm{~V}$ by considering only large size pits. The existence of different $\lambda$ values correlates to different types of pits in the SEM micrographs and can be accounted for by considering that inclusions contribute together with the aggressive anion penetration through passive layer to the pitting corrosion of $316 \mathrm{SS}$. It is known that sulphide inclusions are preferred sites for the nuclcation of corrosion pits on $\mathrm{SS}[20,21]$. In this case, the surface concentration of sulphide inclusions was estimated between 3000 and $5000 \mathrm{~cm}^{-2}$ and the corresponding average radius results close to $1.5 \mu \mathrm{m}$. From the standpoint of the metal structure, the inclusions participating in the pitting process can be distinguished into two different types, namely, those lying part in the passive layer and part in the metal (type I centres), which causes an appreciable modification of the structure and characteristics of the passive layer, and those inclusions located just under the passive layer skin (type II centres), which have a minor influence on the passive layer. At early stages of pitting one should expect that only centres of type 1 become relevant, and therefore it is reasonable that $i_{1}$ values be associated with the attack of the metal at centres of type I. These centres can be completely converted into pits in a short period of time leading to large pits (Fig. 7b). Otherwise, values of $\lambda_{2}$ presumably refer to pitting process at centres of type II, yielding small pits (Fig. 7c) as the kinetics of the process is determined by the lower $\lambda_{2}$ value. Specimens with type I centrcs can also offer type II centres for pitting, this possibility should depend on the proper structure of the passive layer.

In conclusion the pitting corrosion mechanism of $316 \mathrm{SS}$ in neutral buffered solutions containing chloride ions can be described in terms of stochastic and deterministic formalisms depending on the time and potential windows of the experimental approach. In any case the reaction model implies that pit nucleation occurs at centres on the metal surface whose reacting characteristics depend on both the charac- teristics of the passive layer at the points of attack and the presence of inclusions at the metal surface.

Acknowledgements - This work was financially supported by the Consejo Nacional de Investigaciones Científicas y Técnicas and the Comisión de Investigaciones Científicas de la Provincia de Buenos Aires.

\section{REFERENCES}

1. N. Sato, J. electrochem. Soc: 123, 1197 (1976).

2. T. Shibata and T. Takayama, Corrosion 33, 243 (1977).

3. D. E. Williams, C. Westcott and M. Fleischmann, in Passivity of Metals and Semiconductors (Edited by $\mathbf{M}$. Froment), p. 217

4. D. E. Williams, C. Westcott and M. Fleischmann, $J$. electrochem. Soc. 132, 1796 (1985).

5. C. Gabrielli, M. Keddam, M. Krarti and C. Pallotta, 166th Meeting of the Electrochemical Soc., New Orleans (1984).

6. V. Bertocci and Y. Yang-Xiang, J. electrochem. Soc. 131, 1011 (1984).

7. H. Saito, T. Shibata and G. Okamoto, Corros. Sci. 19,693 (1979).

8. G. Okamoto, Corros. Sci. 13, 471 (1973).

9. C. Pallotta, N. de Cristofaro, R. C. Salvarezza and A. J. Arvia, Electrochim. Acta 31, 1265 (1986).

10. N. Ramasubramanian, N. P. Preocanin and R. D. Davison, J. electrochem. Soc. 132, 793 (1985).

11. C. Acosta, R. C. Salvarezza, H. A. Videla and A. J. Arvia, Corros. Sci. 25, 291 (1985).

12. C. A. Acosta, D. V. Vazquez Moll, R. C. Salvarezza, H. A Videla and A. J. Arvia, Corros. Sci. 25, 239 (1985).

13. M. R. G. de Chialvo, D. V. Vazquez Moll, R. C. Salvarezza and A. J. Arvia, Electrachim. Acta 30, 1501 (1985).

14. A. Milchev and V. Tsakova, Electrochim. Acta 30, 133 (1985).

15. I. Ole[jord, B. Brox and V. Jelvestam, J. electrochem. Soc. 132, 2855 (1985).

16. O. Murphy, J. O'M. Bockris and T. E. Pou, J. electrochem. Soc. 130, 1792 (1983).

17. T. Okada, J. electrochem. Soc. 131, 241 (1984).

18. M. Abyaneh and M. Fleischmann, $J$, electroanal. Chem. 119, 187 (1981).

19. V. Bostanov, W. Obretenov, G. Staikov and E. Budevski, J. electroanal. Chem. 146, 303 (1983).

20. N. Sato, J. electrochem. Soc. 129, 261 (1982)

21. P. E. Manning, D. J. Duquette and W. F. Savage, Corrosion 36, 6 (1980). 